Journal of Computer Science 8 (2): 227-231, 2012

ISSN 1549-3636

(C) 2012 Science Publications

\title{
Artificial Bee Colony based Energy Aware and Energy Efficient Routing Protocol
}

\author{
${ }^{1}$ Visu, P., ${ }^{2}$ S. Koteeswaran and ${ }^{3}$ J. Janet \\ ${ }^{1,2}$ Department of CSE, Research Scholar, \\ Vel Tech Dr. RR and Dr. SR Technical University, Chennai, India \\ ${ }^{3}$ Professor and Head Department of CSE, Dr. MGR University, Chennai, India
}

\begin{abstract}
Problem statement: Network becomes very important technology in the day to day life style of modern computer era, in which the wireless communication become very popular for the last few years. In the same manner, the other wireless network, i.e., ado network is very popular among the researchers in the past few decades. Approach: The Ad hoc networks are defined as a multi-hop radio relaying and are capable of operating without the support of any fixed infrastructure. Identification of optimal route for data communication, efficient utilization of energy, clustering, providing congestion free communication, offering scalability, maintaining the Quality of Service (QoS) are few research issues in the Adhoc Network. Results: This study is focusing the review of current research and the requirements, characteristics and design of wireless ado routing protocol. Conclusion: The results of the proposed work show that the proposed swarm intelligence based algorithm will provide optimality than existing systems.
\end{abstract}

Key words: Wireless communication, ado network, energy efficient routing, energy aware routing, routing protocol, swarm intelligence, traveling salesman, mathematical model

\section{INTRODUCTION}

The routing is the problem of finding closed tour which aims for minimal cost that visits each city once, this problem is also called as Traveling Salesman Problem (TSP). The mathematical model for network routing can be expressed in graph theory as:

Let $\mathrm{V}=\{\mathrm{a}, \ldots, \mathrm{z}\}$ be a set of cities

A $\quad=\{(r, s): r, s \in V\}$ be the edge set

and $\delta(\mathrm{r}, \mathrm{s})=\delta(\mathrm{s}, \mathrm{r})$ be a cost measure associated with edge $(r, s) € A$

In this case, the cities $\mathrm{r} € \mathrm{~V}$ are given by their coordinates $\left(\mathrm{x}_{\mathrm{r}}, \mathrm{y}_{\mathrm{r}}\right)$ and $\delta(\mathrm{r}, \mathrm{s})$ is the Euclidean distance between $\mathrm{r}$ and $\mathrm{s}$, then it is Euclidean TSP. If $\delta(\mathrm{r}, \mathrm{s}) \neq$ $\delta(s, r)$ for at least some $(r, s)$ then the TSP becomes an Asymmetric TSP.

Given from the above graph abstraction (Peterson and Davie, 2000), the problem of finding the optimal path in terms of least cost from a source to a destination requires identifying a series of links such that:

- The first link in the path is connected to the source

- The last link in the path is connected to the destination

- For all i, the $\mathrm{i}$ and i-1st link in the path are connected to the same node
- For the least cost path, the sum of the cost of the links on the path is the minimum over all possible paths between the source and destination

- If all link costs are the same, the least cost path is also the shortest path (i.e., the path crossing the smallest number of links between the source and the destination

Quality of Service support for Mobile Ad-hoc Networks is a challenging task due to dynamic topology and limited resource. The main purpose of QoS routing is to find a feasible path that has sufficient resources to satisfy the constraints. A fundamental problem in QoS routing is to find a path between a source and destination that satisfies two or more end-to-end QoS constraints.

The wireless routing is complex than wired routing due to many constraints and more computational effort. Therefore some more tasks in addition to wired network are added to meet the wireless environment. These specific tasks are very important for the design of wireless network routing protocol due to the following requirements:

- Minimization of power requirement

- Utilizing minimal network resources like bandwidth

Corresponding Author: Visu, P., Department of CSE, Vel Tech Dr. RR and Dr. SR Technical University, Chennai, India Tel: 91-9884002675 
- Gathering information and updating link failures

Therefore the traditional routing protocols which applied for wired network such as Open Shortest Path First (OSPF), Routing Information Protocol (RIP) are inefficient for the wireless environment, due to (Manoj and Murthy, 2004), frequent mobility, limited bandwidth and power, hidden and exposed terminal and interference. The highly dynamic nature of mobile network leads to frequent path breaks. Therefore the requirements of the wireless routing protocol must be able to perform an efficient and effective mobility management. As radio band is limited, the bandwidth of wireless network is also limited compared to wired network. Due to frequent changes in topology, maintaining consistent topological information at all the nodes involves greater control overhead. This, in turn, leads to larger bandwidth wastage, reduced network efficiency and performance. The Hidden and exposed terminal problems leads to collision and congestion in the network.

The most common protocols are AODV, DSDV, DSR in ado used to ensure the data transmission among themselves.

\section{MATERIALS AND METHODS}

Wireless ado networks are self-organized dynamic networks can share wireless channel without any established central control standard for IEEE 802.11. In ado network, each mobile node has many limitations like lesser battery power, lower processing power and limited on-board memory. And the ado network will communicate with each other mobile nodes in a multi-hop fashion.

Various routing protocols for MANET is designed to meet these challenges, each of them having their own way of choosing how and when to run neighbor discoveries and compute shortest paths. Designing an efficient routing protocol for ado network is a very challenging task and it has been an active area of research. Many routing protocols have been proposed and these protocols can be broadly classified as proactive and reactive.

Flat protocols: Proactive protocols use the same approach as wired routing, in which the nodes will regularly exchange the routing information and using this data structures decide the optimal route. When a source node wants to send or forward a packet, it only has to read its routing table for sending the data via the mentioned interface in the routing table. It is then easy and fast but the prohibitive bandwidth overhead in order to continuously exchange routing information is pitfall of this method. The exchanged data can take two forms, both used in the wired network: Distance Vector (DV) and Link State (LS). The DV is based on the distributive Bellman-Ford Algorithm and the LV is based on Djickstra shortest path algorithm. The DV is used in MANET in the form Destination-Sequenced Distance-Vector (DSDV) routing. The LS is based on having information about the topology which is then used by the nodes to compute shortest paths. It is used in the Topology dissemination Based on Reverse-Path Forwarding (TBRPF) and Optimized Link-State Routing (OLSR) protocols for MANET.

In order to reduce network traffic allocated to routing information, reactive protocols compute the path to a destination only when needed. It also relies on flooding: when a node wants to send a packet and does not have fresh information about its destination, it broadcasts a request for a path to this destination. The request is flooded and keeps track of the network path followed. The destination and nodes that have a fresh path to this destination respond to the request. The source of the request chooses the best response and caches it. The flooding process operates only on demand, avoiding bandwidth overhead. It does not take care of inactive nor unused nodes. On the other hand, this incurs a latency that may not be acceptable in some contexts. Ad-hoc On-demand Distance Vector (AODV) and Dynamic Source Routing (DSR) protocols are well known examples of reactive protocols

Hybrid protocols: The idea of hybrid protocols is using both proactive and reactive approaches, each one with a different scope. The network is divided into smaller groups (or clusters). Then, a proactive paradigm is used to collect information about nodes within the cluster, while a reactive paradigm is used for communications with nodes in distant clusters. Sending a packet within a cluster (which is supposed to occur more often) is fast and exchanged routing information is still rather small. Sending a packet out of a cluster will probably take longer, but this should happen not as often and the prohibitive bandwidth overhead is avoided. The other side of the coin is a much more complicated design and implementation of such a protocol. The difficult part is deciding how the clusters are formed and how to handle changes in the topology. The Zone Routing Protocol (ZRP) and the Hazy Sighted Link State (HSLS) protocol are two good examples in this category.

Hierarchical routing protocols: Hierarchical protocols use the idea of clustering previously discussed and generalize it to a multilevel hierarchy. Each node belongs to the lowest level. They are divided into clusters that may overlap as in hybrid protocols. But here, nodes within a cluster elect a leader that belongs 
also to the upper level of the hierarchy; the set of leaders is also divided in clusters with their own leader and so forth. Again, choosing the limits of clusters and their leader may be intricate. When a packet is sent, it is forwarded from level to level until it reaches a cluster where a node is a (possibly indirect) leader of the destination. The packet then travels down in the hierarchy until it reaches a level-0 cluster to which the destination belongs.

Geographic routing protocols: Geographic protocols can be used when nodes have knowledge of the location of a given address. Thus they do not need to perform flooding; instead they forward the packet to only one node (or a set of nodes) on the path towards the destination. Simplest protocols use a greedy algorithm to do so: Packets are forwarded to the directly reachable node that is closest to the destination. If the destination cannot be reached with this path, backtracking is used to find another path. The Greedy Perimeter Stateless Routing (GPSR) protocol is an example of geographic protocol.

Recent research: The existing heuristics have addressed only some aspects of MANET characteristics, such as load balancing, mobility, or algorithmic convergence. Therefore Baoa and GarciaLuna-Aceves (2010) introduces a novel approach to solving the connected dominating set election problem, in which the topology management by priority ordering or integrating multiple factors (energy and mobility) into a single metric for cluster election decisions. This approach uses the Neighbor-aware Contention Resolution (NCR) algorithm to provide fast convergence and load balancing with regard to the battery life and mobility of mobile nodes. Based on NCR, the authors assign randomized priorities to mobile stations and elect a Minimal Dominating Set (MDS) and the Connected Dominating Set (CDS) of an ad hoc network according to these priorities. In doing so, the method proposes in this study, called TMPO, which requires only two-hop neighbor information for the MDS elections. The dynamic priorities assigned to nodes are derived from the node identifiers and their "willingness' to participate in the backbone formations. The willingness of a node is a function of the mobility and battery life of the node. The integrated consideration of mobility, battery life and deterministic node priorities makes TMPO one of the best performing heuristics for topology management in ad hoc networks.

Swarm intelligence based routing: In the recent network routing, Ant-Net Routing using Ant Colony Optimization (ACO) technique provide a better result than others due to its real time computation and less Control Overhead (Dorigo et al., 1996; 2004; Dorigo and Gambardella, 1997; Sim and Sun, 2003) comparing all routing algorithms with ACO, concludes that ants are relatively small, can be piggybacked in data packets and more frequent transmission of ants may be possible in order to provide updates of routing information for solving link failures. Hence, using ACO for routing in dynamic network seems to be appropriate. Routing in ACO is achieved by transmitting ants rather than routing tables or by flooding LSPs. Even though it is noted that the size of an ant may vary in different systems/implementations, depending on their functions and applications, in general, the size of ants is relatively small, in the order of 6 bytes.

\section{RESULTS}

Swarm intelligence is a new discipline of study that contains a relatively optimal approach for problem solving which are the imitations inspired from the social behavior of insects and animals, for example, Ant Colony Optimization (ACO) algorithm, Honey Bee Algorithms, Fire Fly Algorithm. The "ACO Algorithm' ' is a study derived from the observation of real ants' behavior and uses these models as a source of inspiration for the design of novel algorithms, which is the solution for optimization and distributed control problems. The Honey Bee Mating algorithm is the growing technique, which is proposed in late 2005, for many engineering applications.

To identify the optimal location of bio-mass power plant (Veraa et al., 2010), Resource Allocation (Quijanoa and Passino, 2010), Constraint Optimization Problem (Karaboga and Akay, 2009), data Clustering in data mining (Karaboga and Ozturk, 2011) are some of the successful solutions based on $\mathrm{ABC}$ algorithm. The detailed honey bee mating algorithm is explained.

Mathematical model of ABC algorithm: The algorithm requires a number of parameters to be set, namely: number of scout bees (n), number of elite bees (e), number of patches selected out of $n$ visited points (m), number of bees recruited for patches visited by "elite bees" (nep), number of bees recruited for the other (m-e) selected patches (nsp), size of patches (ngh) and stopping criterion. The algorithm starts with the $\mathrm{n}$ scout bees being placed randomly in the search space.

The bees search for food sources in a way that maximizes the ratio Eq. 1:

$$
F(\theta)=\frac{E}{T}
$$


Where, $\mathrm{E}$ is the energy obtained and $\mathrm{T}$ is the time spent for foraging. Here $\mathrm{E}$ is proportional to the nectar amount of food sources.

In a maximization problem, the goal is to find the maximum of the objective function $F(\theta), \theta \in R^{P} \cdot R^{P}$ represents the region of search area.

Assume that $\theta_{\mathrm{i}}$ is the position of the $\mathrm{i}^{\text {th }}$ food source; $\mathrm{F}\left(\theta_{\mathrm{i}}\right)$ represents the nectar amount of the food source located at $\theta_{\mathrm{i}}$ and it is proportional to the energy $\mathrm{E}\left(\theta_{\mathrm{i}}\right)$.

\section{DISCUSION}

Let $\mathrm{P}(\mathrm{C})=\left\{\theta_{\mathrm{i}}(\mathrm{C}) \mid \mathrm{i}=1,2 \ldots \mathrm{S}\right\}$ represent the population of food sources being visited by bees, in which, $\mathrm{C}$ is cycle and $\mathrm{S}$ is number of food sources around the hive. The preference of a food source by the worker bee depends on the nectar amount $F(\theta)$ of that food source. As the nectar amount of the food source increases, the probability with the preferred source by the worker bee increases proportionally. Therefore, the probability with the food source located at $\theta_{\mathrm{i}}$ will be chosen by a bee can be expressed as Eq. 2:

$$
P_{i}=\frac{F\left(\theta_{i}\right)}{\sum_{k=1}^{s} F\left(\theta_{k}\right)}
$$

The position of the selected neighbour food source is calculated as the following Eq. 3:

$\theta_{\mathrm{i}}(\mathrm{C}+1)-\theta_{\mathrm{i}}(\mathrm{C})$

And the stop criteria of the system are Eq. 4:

$\mathrm{N}_{\mathrm{i}}(\mathrm{Q})-\mathrm{N}_{\mathrm{i}}(\mathrm{E})^{3} \mathrm{H}_{\mathrm{th}}$

Where:

$N_{i}(Q)=$ The values of nectar of Queen

$\mathrm{N}_{\mathrm{i}}(\mathrm{E})=$ The values of nectar of Elite bee

$\mathrm{H}_{\mathrm{th}}=$ The minimum threshold value of the Hive

At the end of iteration, the colony will have two parts to its new population-representatives from each selected patch and other scout bees assigned to conduct random searches. More detailed study on swarm intelligence is available in Chandra (Mohan et al., 2008; Mohan and Baskaran, 2011a; 2011b).

\section{CONCLUSION}

In wireless network, variety of routing issues is resolved based on various routing metric in the past few decades. For example, the communication overhead is taken as major issue by Natsheh and Buragga (2010), in which, broadcasting algorithm is improved under high mobility and density through the routing process. Banerjee and Dutta (2010) proposed Link Stability and Node Energy Conscious (LSNEC). In which AODV and Associatively Based Routing (ABR) is improved using Quick Local Repair scheme (QLR). Kaabneh et al. (2009) proposed pure flooding based routing technique for mobile adhoc network, which offers reliability and link capability.

The objective of this study is to briefly review the major contribution in the adhoc routing protocols. In order to meet the objective, this study initially provides the introduction of the wired routing and explained about the wired routing in terms of mathematical model. The dissimilarity of wired and wireless routing is explained and in the consequence, the methodology of adhoc routing is reviewed. The objective of the study is fulfilled by providing necessary and limited information regarding the adhoc routing which also will guide the new researcher. This study also provides the recent research proposals like ant colony optimization and artificial bee colony algorithms based wireless routing protocols.

\section{REFERENCES}

Banerjee, A. and P. Dutta, 2010. Link stability and node energy conscious local route-repair scheme for mobile ad hoc networks. Am. J. Applied Sci., 7: 1139-1147. DOI: 10.3844/ajassp.2010.1139.1147

Baoa, L. and J.J. Garcia-Luna-Aceves, 2010. Stable energy-aware topology management in ad hoc networks. Ad Hoc Netw. 8: 313-327. DOI: 10.1016/j.adhoc.2009.09.002

Dorigo, M. and L.M. Gambardella, 1997. Ant colony system: A cooperative learning approach to the traveling salesman problem. IEEE Trans. Evol. Comput., 1: 53-66. DOI: 10.1109/4235.585892

Dorigo, M., M. Birattari and T. Stutzle, 2004. Ant colony optimization. IEEE Comput. Intell. Mag., 1: 28-39. DOI: 10.1109/MCI.2006.329691

Dorigo, M., V. Maniezzo and A. Colorni, 1996. Ant system: Optimization by a colony of cooperating agents. IEEE Trans. Syst. Man Cybernetics, 26: 2941. DOI: $10.1109 / 3477.484436$

Kaabneh, K., A. Halasa and H. Al-Bahadili, 2009. An effective location-based power conservation scheme for mobile ad hoc networks. Am. J. Applied Sci., 6: 1708-1713. DOI: 10.3844/ajassp.2009.1708.1713 
Karaboga, D. and B. Akay, 2009. A comparative study of Artificial Bee Colony algorithm. Applied Math. Comput., 214: 108-132. DOI: 10.1016/j.amc.2009.03.090

Karaboga, D. and C. Ozturk, 2011. A novel clustering approach: Artificial Bee Colony (ABC) algorithm. Applied Soft Comput., 11: 652-657. DOI: 10.1016/j.asoc.2009.12.025

Manoj, B.S. and C.S.R. Murthy, 2004. Ad Hoc Wireless Networks: Architectures and Protocols. 1st Edn., Pearson Education India, India, ISBN-10: 8131706885, pp: 878.

Mohan, B.C. and R. Baskaran, 2011a. Survey on recent research and implementation of ant colony optimization in various engineering applications. Int. J. Comput. Intell. Syst., 4: 566-582.

Mohan, B.C. and R. Baskaran, 2011b. A survey: Ant colony optimization based recent research and implementation on several engineering domain. Exp. Syst. Appli., 39: 4618-4627. DOI: 10.1016/j.eswa.2011.09.076

Mohan, B.C., R. Sandeep and D. Sridharan, 2008. A data mining approach for predicting reliable path for congestion free routing using self-motivated neural network. Stud. Comput. Intell., 149: 237246. DOI: $10.1007 / 978-3-540-70560-4 \_20$
Natsheh, E. and K. Buragga, 2010. Density based routing algorithm for spare/dense topologies in wireless mobile ad-hoc networks. Am. J. Eng. Applied Sci., 3: 312-319. DOI: 10.3844/ajeassp.2010.312.319

Peterson, L.L. and B.S. Davie 2000. Computer Networks: A Systems Approach. 2nd Edn., Morgan Kaufmann, San Fransisco, Beijing, ISBN: 7111075269 , pp: 748.

Quijanoa, N. and K.M. Passino, 2010. Honey bee social foraging algorithms for resource allocation: Theory and application. Eng. Appli. Artif. Intell., 23: 845861. DOI: 10.1016/j.engappai.2010.05.004

Sim, K.M. and W.H. Sun, 2003. Ant colony optimization for routing and load-balancing: survey and new directions. IEEE Trans. Syst. Man Cybernetics, 33: 560-572. DOI: 10.1109/TSMCA.2003.817391

Veraa, D., J. Carabiasb, F. Juradoa, N. Ruiz-Reyes, 2010. A honey bee foraging approach for optimal location of a biomass power plant. Applied Energy, 87: 2119-2127. DOI: $10.1016 /$ j.apenergy.2010.01.015 